\title{
Article \\ Sleep Disordered Breathing, Obesity and Atrial Fibrillation: A Mendelian Randomisation Study
}

\author{
Maddalena Ardissino ${ }^{1,2, \dagger}$, Rohin K. Reddy ${ }^{1,+}{ }^{\circledR}$, Eric A. W. Slob ${ }^{3}$, Kiran H. K. Patel ${ }^{1}$, David K. Ryan ${ }^{4,5}$, \\ Dipender Gill $4,5,6,7, \neq\left(\mathbb{D}\right.$ and Fu Siong $\mathrm{Ng}^{1, *, \neq \mathbb{C}}$
}

1 National Heart and Lung Institute, Imperial College London, London W12 0NN, UK; maddalena.ardissino13@imperial.ac.uk (M.A.); rohin.reddy17@imperial.ac.uk (R.K.R.); kiran.patel@imperial.ac.uk (K.H.K.P.)

2 Nuffield Department of Population Health, Old Road Campus, University of Oxford, Oxford OX3 7LF, UK

3 MRC Biostatistics Unit, School of Clinical Medicine, University of Cambridge, Cambridge CB2 0SR, UK; eric.slob@mrc-bsu.cam.ac.uk

4 Clinical Pharmacology Group, Pharmacy and Medicines Directorate, St George's University Hospitals NHS Foundation Trust, London SW17 0QT, UK; davidkdryan@gmail.com (D.K.R.); dipender.gill@imperial.ac.uk (D.G.)

5 Clinical Pharmacology and Therapeutics Section, Institute for Infection and Immunity, St George's, University of London, London SW17 ORE, UK

6 Department of Epidemiology and Biostatistics, School of Public Health, Imperial College London, London W2 1PG, UK

7 Novo Nordisk Research Centre Oxford, Old Road Campus, Oxford OX3 7FZ, UK

* Correspondence: f.ng@imperial.ac.uk; Tel.: +44-20-7594-3614

+ These authors contributed equally to this work, and are joint first authors.

$\ddagger$ These authors contributed equally to this work, and are joint senior authors.

Citation: Ardissino, M.; Reddy, R.K.; Slob, E.A.W.; Patel, K.H.K.; Ryan, D.K.; Gill, D.; Ng, F.S. Sleep Disordered Breathing, Obesity and Atrial Fibrillation: A Mendelian Randomisation Study. Genes 2022, 13, 104. https://doi.org/10.3390/ genes13010104

Academic Editor: Mikko P. Turunen

Received: 1 December 2021

Accepted: 29 December 2021

Published: 2 January 2022

Publisher's Note: MDPI stays neutral with regard to jurisdictional claims in published maps and institutional affiliations.

Copyright: () 2022 by the authors Licensee MDPI, Basel, Switzerland. This article is an open access article distributed under the terms and conditions of the Creative Commons Attribution (CC BY) license (https:// creativecommons.org/licenses/by/ $4.0 /)$.

\begin{abstract}
It remains unclear whether the association between obstructive sleep apnoea (OSA), a form of sleep-disordered breathing (SDB), and atrial fibrillation (AF) is causal or mediated by shared comorbidities such as obesity. Existing observational studies are conflicting and limited by confounding and reverse causality. We performed Mendelian randomisation (MR) to investigate the causal relationships between SDB, body mass index (BMI) and AF. Single-nucleotide polymorphisms associated with SDB $(n=29)$ and BMI $(n=453)$ were selected as instrumental variables to investigate the effects of SDB and BMI on AF, using genetic association data on 55,114 AF cases and 482,295 controls. Primary analysis was conducted using inverse-variance weighted MR. Higher genetically predicted SDB and BMI were associated with increased risk of AF (OR per log OR increase in snoring liability 2.09 (95\% CI 1.10-3.98), $p=0.03$; OR per 1-SD increase in BMI 1.33 (95\% CI 1.24-1.42), $p<0.001)$. The association between SDB and AF was not observed in sensitivity analyses, whilst associations between BMI and AF remained consistent. Similarly, in multivariable MR, SDB was not associated with AF after adjusting for BMI (OR 0.68 (95\% CI 0.42-1.10), $p=0.12$ ). Higher BMI remained associated with increased risk of AF after adjusting for OSA (OR 1.40 (95\% CI 1.30-1.51), $p<0.001$ ). Elevated BMI appears causal for AF, independent of SDB. Our data suggest that the association between SDB, in general, and AF is attributable to mediation or confounding from obesity, though we cannot exclude that more severe SDB phenotypes (i.e., OSA) are causal for AF.
\end{abstract}

Keywords: atrial fibrillation; sleep-disordered breathing; obstructive sleep apnoea; obesity; Mendelian randomization

\section{Introduction}

Current clinical guidelines support an integrated approach to atrial fibrillation (AF) management, encompassing anticoagulation, symptom management and risk factor optimisation, with recent focus on obesity and obstructive sleep apnoea (OSA) as two reversible risk factors [1]. Sleep-disordered breathing (SDB) is a general term describing difficulties in 
breathing during sleep ranging from snoring to OSA, which is increasing in prevalence $[2,3]$. OSA is the most common form of SDB in the general population, characterised by hypopnoeic and apnoeic collapses of the upper airway during sleep despite ongoing respiratory effort [2]. Based on several observational studies showing an association between OSA with higher rates of $\mathrm{AF}$ [4-6] and observational meta-analysis associating continuous positive airway pressure (CPAP) treatment with reductions in AF recurrence [7], previous clinical guidelines for AF contained a Class IIa recommendation that OSA treatment should be optimised to reduce AF recurrence and improve treatment [1].

However, more recent clinical guidelines have downgraded that recommendation in favour of a Class IIb recommendation that OSA management may be considered to reduce AF incidence, progression, recurrence and symptoms [1]. This reflects a subsequent appreciation of the uncertainty in the evidence base due to the dearth of randomised controlled trial (RCT) evidence and the lack of consensus from observational data. Some large observational studies have failed to demonstrate an association between OSA and AF that is independent of other cardiovascular risk factors $[8,9]$. Furthermore, the presence of OSA, and its treatment with CPAP, have recently been shown not to impact arrhythmia outcomes following ablation [10,11] or cardioversion [10]. Early and small RCTs have shown no impact of CPAP on time to AF recurrence post-cardioversion [12] or differences in AF burden or quality of life [13].

Complicating interpretation of the data on $\mathrm{SDB} / \mathrm{OSA}$ and $\mathrm{AF}$ is the strong association of both SDB/OSA and AF with obesity, which may act as a confounder. Obesity has been shown to be associated with AF [14], with the effects thought to be mediated in part by electrotonic and paracrine effects of epicardial fat [15]. Obesity and SDB are also closely related, whereby obesity both predisposes to, and potentiates SDB [16,17], and developing SDB is associated with subsequent weight gain [18]. With currently available retrospective observational data, it is difficult to determine whether the relationship between SDB/OSA and $\mathrm{AF}$ is mediated or confounded through obesity and raised body mass index (BMI), or whether OSA causes AF directly through independent pathways.

Mendelian randomisation (MR) utilises genetic variants in instrumental variable analysis to investigate relationships between modifiable risk factors and outcomes using observational data [19]. Leveraging genetic variants that are independently and randomly inherited as proxies for modifiable exposures allows for causal inference concerning outcomes. In this way, MR can potentially overcome limitations associated with classical observational epidemiology, namely confounding and reverse causality [19], and may permit delineation of complex pathways relating SDB, BMI and AF. A recent univariable MR study reported a causal association between five single-nucleotide polymorphisms (SNPs) associated with OSA and AF [20]. However, some of the employed OSA instruments were also associated with BMI or whole-body, fat-free mass at the genome-wide significance level, raising the question of whether this relationship was partially mediated via obesity. We therefore applied the multivariable MR paradigm to investigate the relationship between genetically predicted $\mathrm{SDB}$ and $\mathrm{BMI}$ on $\mathrm{AF}$, and to explore the presence of genetic evidence supporting a direct causal effect of SDB in the development of AF independent of BMI.

\section{Materials and Methods}

\subsection{Ethical Approval, Data Availability and Reporting}

All data used for this study are publicly available and their original studies are cited. All these studies obtained relevant participant consent and ethical approval. The paper is reported on the basis of recommendations by The Strengthening the Reporting of Observational Studies in Epidemiology-Mendelian randomization (STROBE-MR) guidelines [21].

\subsection{Data Sources}

For the primary analyses, genetic association estimates for BMI were obtained from the Genetic Investigation of Anthropometric Traits (GIANT) consortium summary statistics [22] 
on patients of European ancestry. Genetic association estimates for snoring obtained from the GWAS performed by Campos et al. [23] on European ancestry individuals were used as a proxy for SDB in general. The trait with the highest genetic correlation with snoring was sleep apnoea $\left(\mathrm{rG}=0.78, \mathrm{SE}=0.17, p\right.$-value $=3 \times 10^{-5}\left(\mathrm{LDSC} \chi^{2}\right.$-test $\left.)\right)$. These analyses suggest that the SNPs for snoring studied are an appropriate and robust surrogate for genetically proxied SDB, and, henceforth, we will refer to the snoring exposure as SDB. Genetic association estimates for AF were obtained from the GWAS by Roselli et al. [24] on 55,114 cases and 482,295 European controls. Summaries of population characteristics for each of these studies are available in the original publications.

\subsection{Instrumental Variable Selection}

To estimate the effect of genetically predicted probable SDB and BMI, respectively, on $\mathrm{AF}, \mathrm{SNPs}$ were selected if they had been associated with snoring or BMI in the respective data source studies at genome-wide significance $\left(p<5 \times 10^{-8}\right)$. Furthermore, SNPs were selected if they were in pair-wise linkage disequilibrium (LD) with $r^{2} 0.001$. Clumping was performed using the TwoSample MR package in R [25]. This resulted in 29 genome-wide significant SNPs for SDB and 453 genome-wide significant SNPs for BMI.

\subsection{Statistical Power}

Statistical power calculations for MR analyses were conducted using the online tool "mRnd calculator" [26] to estimate the minimum effects that we had at least $80 \%$ power to detect.

\subsection{Statistical Analysis}

The flowchart for the statistical analysis plan is displayed in Figure 1. Inverse-variance weighted (IVW) MR with multiplicative random effects was used as the primary analysis method [27]. The IVW MR approach assumes that genetic instruments for each risk factor satisfy the instrumental variable assumptions, which include the assumption that the instrumental variables are not associated with confounder traits of the association between the risk factor and the outcome of $\mathrm{AF}$, and that the instrumental variables are only associated with $\mathrm{AF}$ through their association with the risk factor. The situation where, rather than acting solely through the genetically predicted risk factor of interest, genetic variants have effects on multiple risk factors influencing multiple parallel biological pathways and subsequent phenotypes is termed horizontal pleiotropy and constitutes an important potential violation of the instrumental variable assumptions [19].

To attempt to correct for any potential violations of the assumptions, we used MREgger regression [28], weighted median MR [29] and MR-PRESSO [30] as sensitivity analyses. We opted for these three analyses as they operate in different ways and rely on different assumptions for valid inferences to assess the reliability of MR analyses [31-33].

To investigate an effect of SDB on AF that is not mediated by BMI (and vice versa), summary data multivariable MR was performed. In this analysis, the variant-outcome genetic association estimates are regressed on the variant-exposure and variant-mediator estimates, weighted for the precision of the variant-outcome association, and with the intercept fixed to zero.

All analyses were performed using the MendelianRandomization [34] and TwoSample MR package in R version 4.0.4 [25]. M.A. had full access to all data in the study and takes responsibility for its integrity and data analysis.

\section{Results}

\subsection{Statistical Power}

The univariable MR analysis for BMI and AF had at least $80 \%$ power to detect AF ORs lower than 0.97 and higher than 1.04 per 1-SD kg $/ \mathrm{m}^{2}$ increase in BMI. The univariable MR analysis for SDB and AF had at least $80 \%$ power to detect AF ORs lower than 0.97 and higher than 1.03 per log OR increase in genetically predicted OSA. 

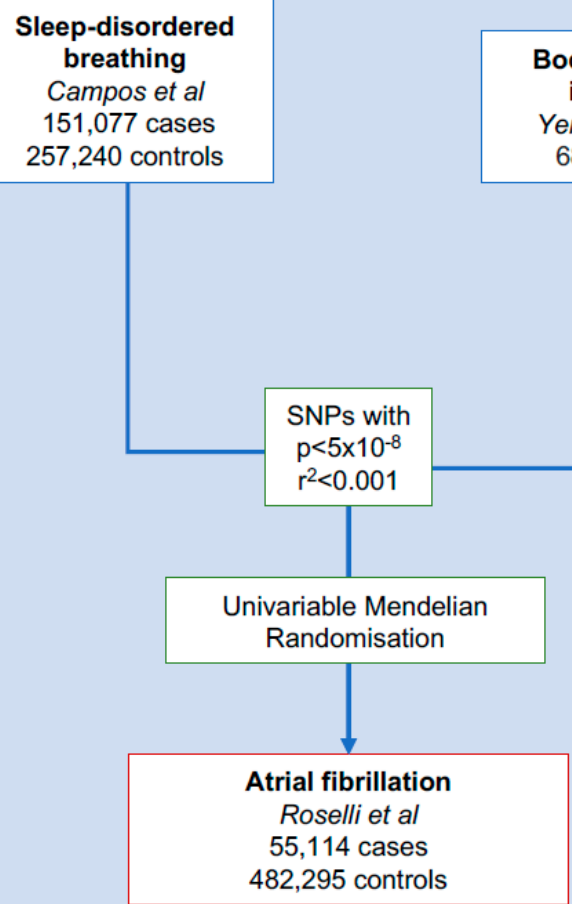

Stage 1

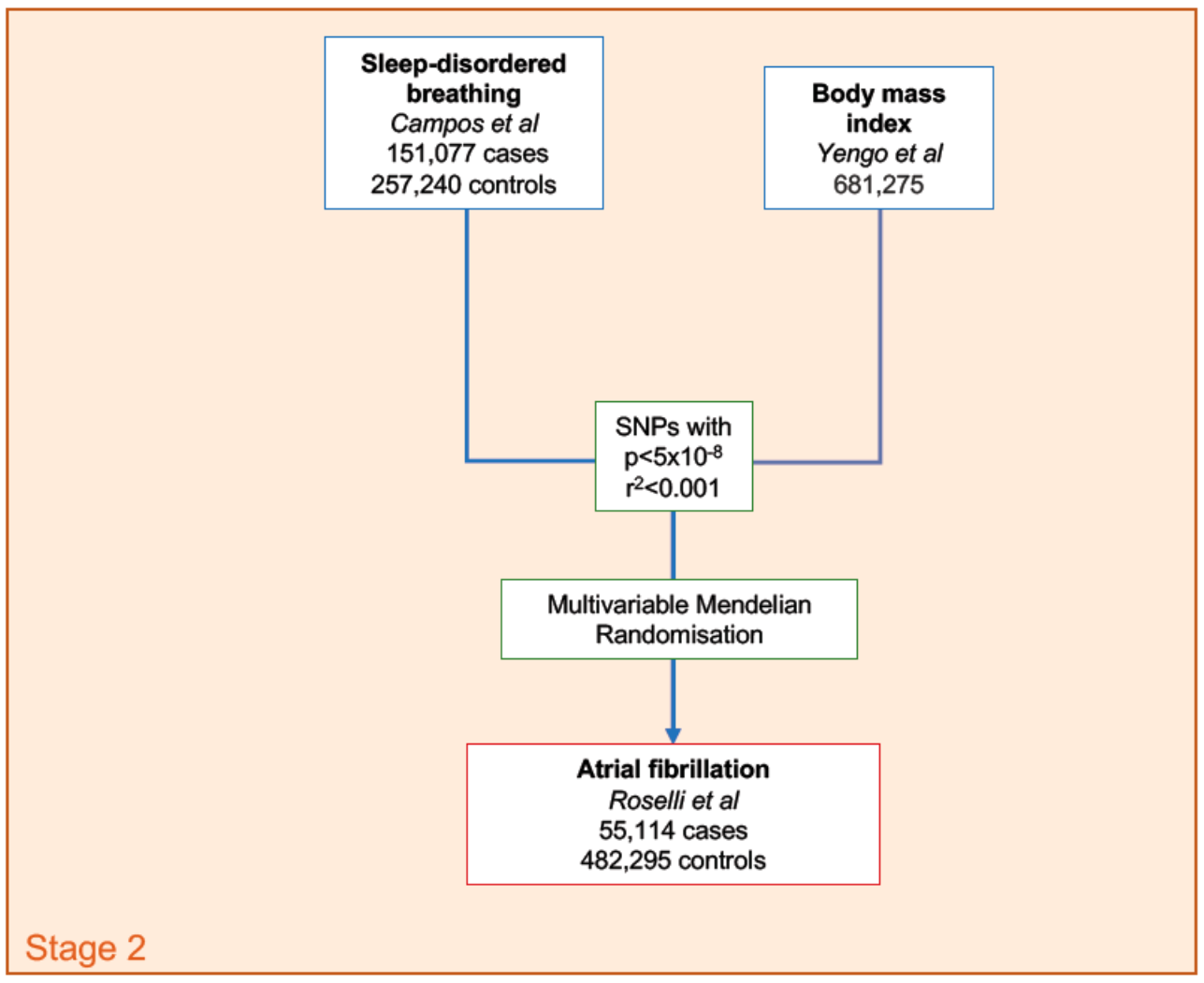

Figure 1. Data acquisition and statistical analysis flowchart.

\section{2. $M R: S D B$ and $A F$}

In IVW MR analysis, higher genetically predicted SDB was associated with increased risk of AF, OR $2.09(95 \%$ CI 1.10-3.98, $p=0.03)$ per log OR increase in snoring liability. There 
was no significant evidence of pleiotropy (MR-Egger intercept 0.02, standard error (SE) 0.01, $p=0.20$ ), but the significant association was no longer observed in MR-Egger (OR 0.24, 95\% CI 0.01-6.53, $p=0.40$ ), weighted median MR (OR 1.40, 95\% CI 0.72-2.70, $p=0.32$ ) analyses or MR-PRESSO (OR 1.85, 95\% CI 1.00-3.41, $p=0.06$, three SNPs excluded).

In multivariable MR analysis, genetically predicted SDB was not associated with AF after adjusting for genetically predicted BMI (OR $0.68,95 \%$ CI $0.42-1.10, p=0.12$ ). The results are summarised in Figures 2 and 3, with complete results displayed in Table S1. SNP MR estimates in the analysis of SDB and AF are displayed in Table S2.
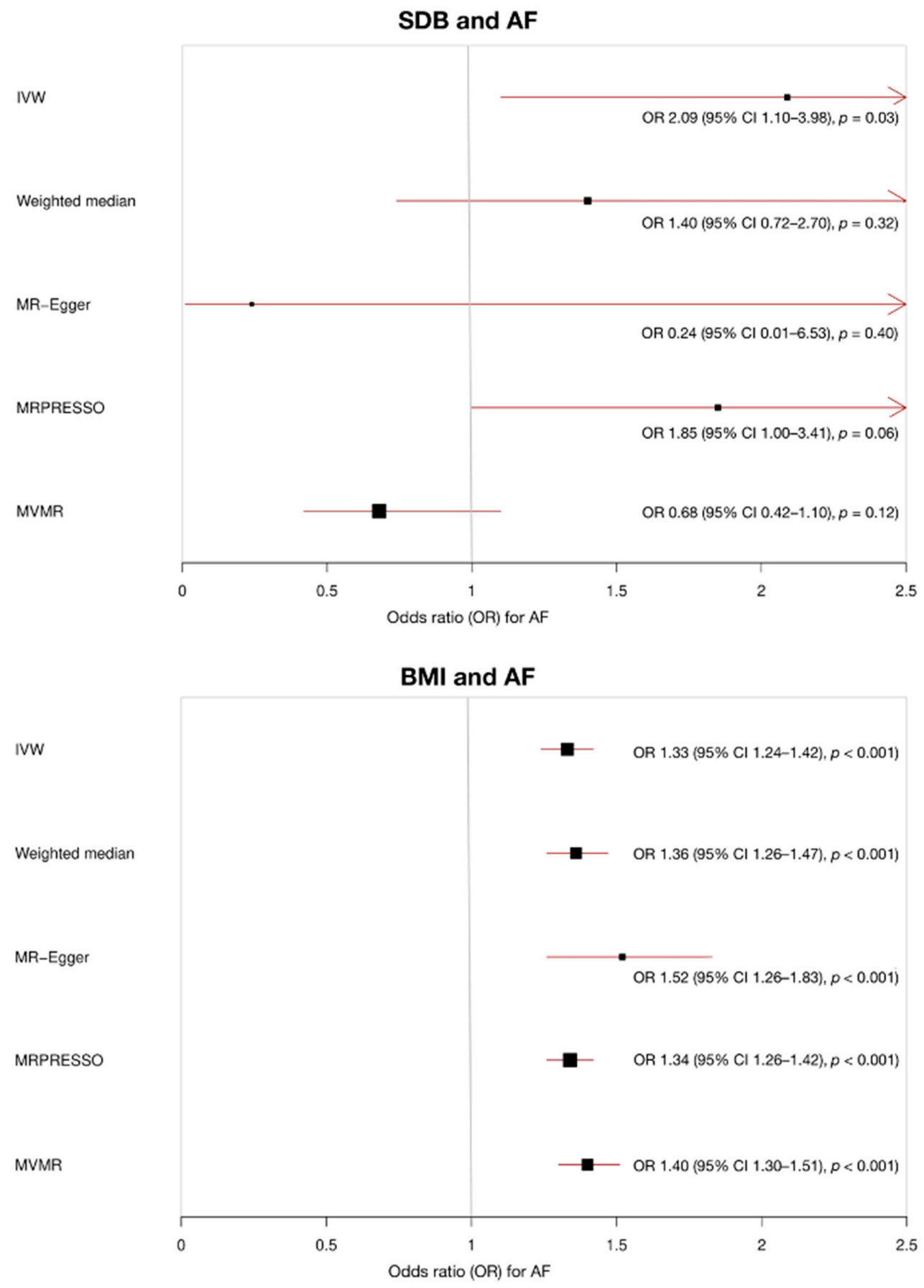

Figure 2. Mendelian randomisation (MR) results for the association of sleep-disordered breathing (SDB) and body mass index (BMI) with atrial fibrillation (AF). When SDB is the exposure (units of log odds ratio snoring liability), multivariable MR analysis adjusts for genetically predicted BMI. When $\mathrm{BMI}$ is the exposure (units of $\mathrm{kg} / \mathrm{m}^{2}$ ), multivariable MR analysis adjusts for genetically predicted SDB. 


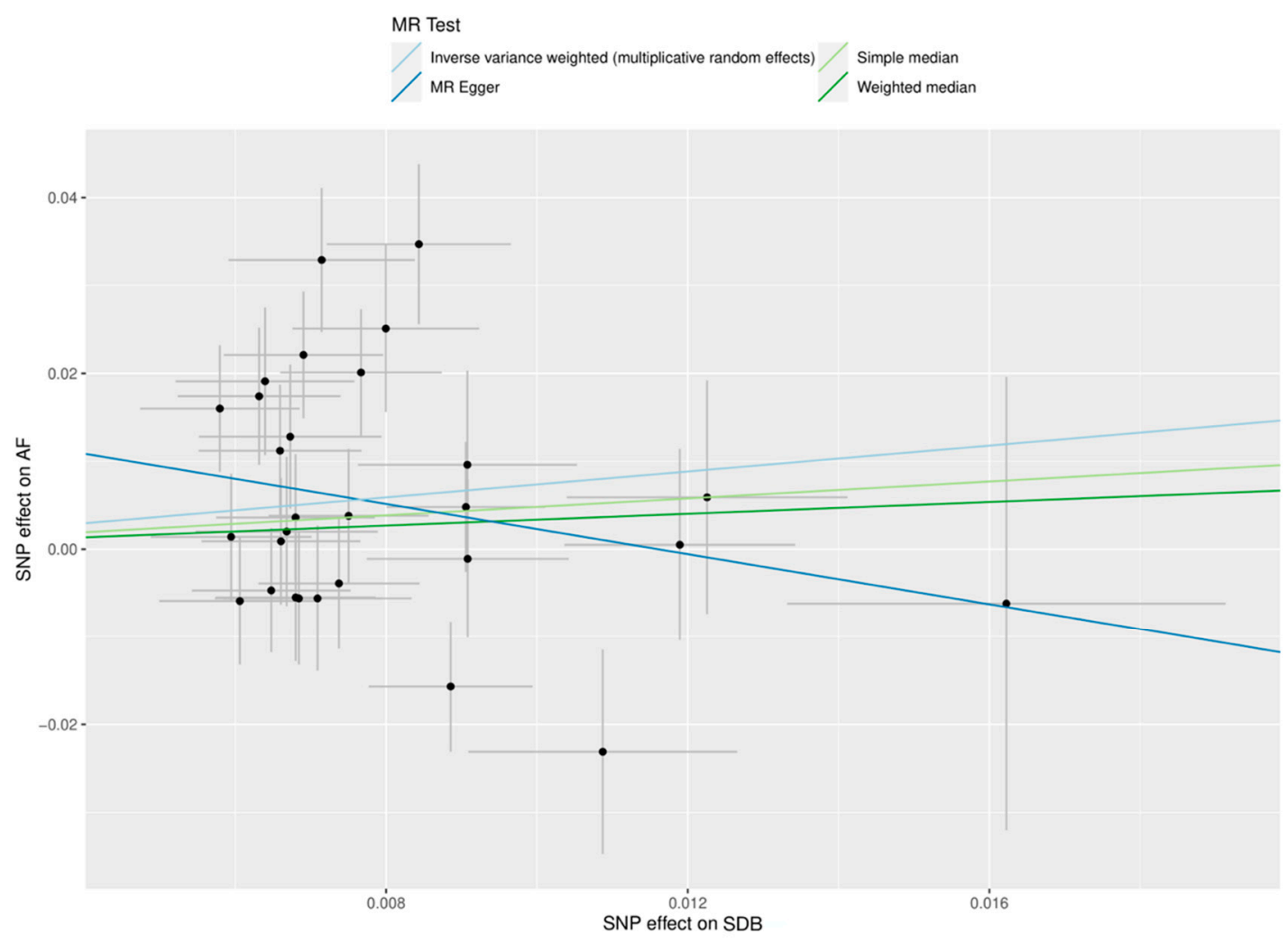

Figure 3. Mendelian randomisation (MR) scatter plot of genetic associations between sleep-disordered breathing (SDB) and atrial fibrillation (AF). The genetic association and corresponding 95\% confidence interval (CI) for each instrumented single-nucleotide polymorphism $(n=29)$ with SDB (x-axis, units of $\log$ odds ratio snoring liability) and AF (y-axis, units of log odds ratio atrial fibrillation liability) are plotted. The gradient of each line represents the MR estimate for the corresponding model.

\section{3. $M R$ : BMI and $A F$}

There was consistent evidence of an association between genetically predicted BMI and AF across the IVW, MR-Egger and weighted median MR analyses. For each $1-S D \mathrm{~kg} / \mathrm{m}^{2}$ increase in genetically predicted BMI, the MR IVW estimate identified increased risk of AF, OR 1.33 (95\% CI 1.24-1.42, $p<0.001)$. There was no evidence of pleiotropy (MREgger intercept $-2 \times 10^{-3}$, SE $\left.1 \times 10^{-3}, p=0.14\right)$, and the association estimate remained consistent in MR-Egger (OR 1.52, 95\% CI 1.26-1.83, $p<0.001$ ), weighted median MR (OR $1.36,95 \%$ CI 1.26-1.47, $p<0.001$ ) and MR-PRESSO (OR 1.34, 95\% CI 1.26-1.42, $p<0.001$, 10 SNPs excluded) sensitivity analyses.

Multivariable MR identified an association between genetically predicted BMI and AF even after adjusting for genetically predicted SDB (OR 1.40, 95\% CI 1.30-1.51, $p<0.001$ ). The results are summarised in Figures 2 and 4, with complete results displayed in Table S1. SNP MR estimates in the analysis of BMI and AF are displayed in Table S3.

\section{Discussion}

In this study, we leveraged genetic data to investigate the complex relationship between SDB, BMI and AF in a two-sample MR study. In univariable MR analysis, genetically predicted SDB was associated with increased risk of AF, though this relationship was lost in sensitivity analyses more robust to the presence of pleiotropic and outlying variants. Using multivariable MR analysis adjusting for genetically predicted BMI, our results suggest SDB, in general, is unlikely to be causally related to the development of AF through pathways, independent of BMI. The role of BMI in mediating the relationship between SDB and AF is highlighted by the IVW MR effect estimate becoming non-significant following inclusion of BMI in the multivariable MR model. We also show that BMI is likely to be causally 
related to $\mathrm{AF}$, independent of SDB, even when accounting for pleiotropic or outlier SNPs. Taken together, these results suggest that BMI is a modifiable risk factor for AF while the association between SDB, in general, and AF is likely to be attributable to mediation or confounding from BMI.

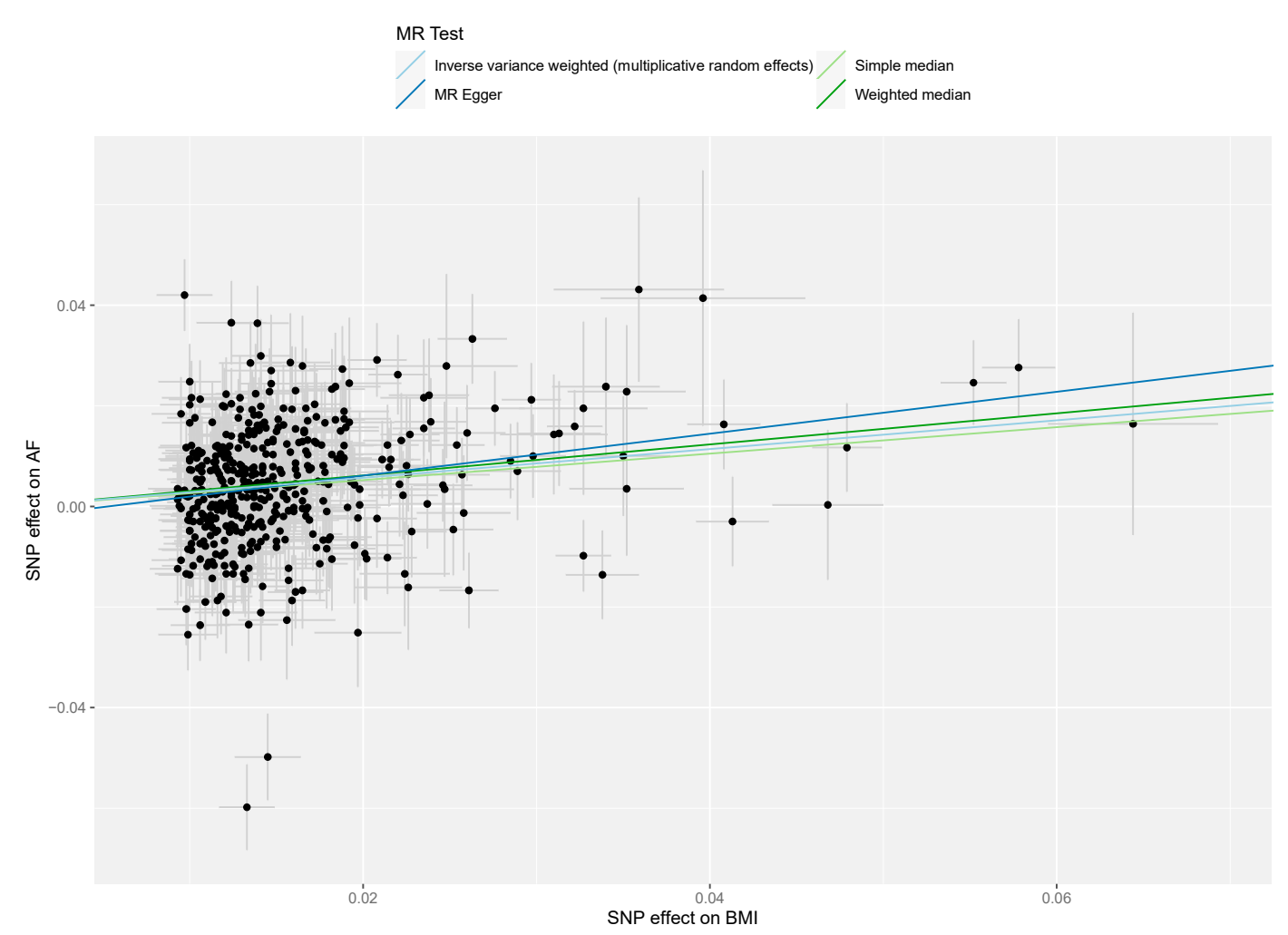

Figure 4. Mendelian randomisation (MR) scatter plot of genetic associations between body mass index (BMI) and atrial fibrillation (AF). The genetic association and corresponding $95 \%$ confidence interval (CI) for each instrumented single-nucleotide polymorphism $(n=453)$ with BMI (x-axis, units of $\mathrm{kg} / \mathrm{m}^{2}$ ) and AF (y-axis, units of log odds ratio atrial fibrillation liability) are plotted. The gradient of each line represents the MR estimate for the corresponding model.

\subsection{BMI Is Causally Associated with AF}

The present study further strengthens the evidence base for the causal role of obesity on AF development and confirms the results of previous MR analyses [35,36]. Obesity is a rising global health challenge that is associated with significant indirect and direct adverse cardiometabolic effects. High BMI is associated with atrial myocardial remodelling [37], atrial fibrosis [37] and epicardial fat deposition [15]. Epicardial fat is a highly pro-arrhythmic entity causing abnormal conduction and repolarisation via paracrine mechanisms and direct fatty myocardial infiltration [15]. The findings of this study, therefore, corroborate current knowledge on the strength of the association between high BMI and AF. Clinically, these results are supported by randomised data. An RCT investigating an intensive structured weight loss programme resulted in reductions in AF burden and severity [38]. In a population with persistent AF, the RACE-3 trial showed improvements in maintenance of sinus rhythm with an intervention of physical activity and dietary restrictions, alongside optimised medical therapy [39]. Altogether, our study provides further evidence to support the strength of this pathological pathway and highlights the paramount importance of risk factor modification through weight loss.

\subsection{SDB Is not Causal for AF Independent of BMI}

The results of this study did not support a causal pathway between SDB, in general, and AF, after accounting for BMI. There is a growing body of observational [8-11] and 
clinical trial evidence [12,13] suggesting a possible lack of direct causal association between OSA, the most common form of SDB, and AF. A recent, small RCT failed to show a benefit of CPAP against AF recurrence after cardioversion in patients with persistent AF [12], though the results should be interpreted with caution as recruitment targets were not met. Another adequately powered trial enrolling patients with paroxysmal AF failed to show a benefit of CPAP in reducing AF burden in patients with moderate to severe OSA [13]. However, there are also many studies that support an independent association between OSA and AF. Experimental models of OSA have previously shown increased vulnerability to AF [40]. An association between OSA and increased risk of AF in community and sleep clinic cohorts has been reported in observational studies [4-6] and observational metaanalysis reported reduced AF recurrence with CPAP therapy [7]. Similarly, a recent MR study reported a Mendelian randomisation association between genetically proxied OSA and AF, though only five SNPs were instrumented in this analysis, with two associated with BMI or whole-body, fat-free mass at the genome-wide significance level [20]. Our study builds on these data by including almost six-fold more SNPs significantly correlated with sleep apnoea traits, in addition to performing multivariable MR to control for the well-characterised, powerful pleiotropic effects of BMI on the relationship between SDB and AF.

A possible explanation to reconcile our findings with the observational studies supporting a causal link between OSA and AF is that statistical adjustment for confounders in observational studies is limited by unmeasured or unmeasurable confounding, to which MR analysis is more robust. Furthermore, multivariable regression can be limited by violation of the assumption that no included covariates are subject to collider bias or measurement imprecision [41]. Considering BMI as a covariate, if BMI measurements were subject to random error or intraindividual variation, regression coefficients would be attenuated such that the impact of BMI on the relationship between OSA and AF would be underestimated. Genetic variants strongly related with OSA and BMI generally maintain their associations throughout life, enabling the MR approach to reduce attenuation by errors and resultant bias [19]. Finally, our study looked as SDBs, in general, as the exposure, rather than OSA specifically. It is possible that the more severe forms of SDB, such as OSA, are causally associated with AF, and this effect was diluted and lost when considering all SDBs, in general. Larger future GWAS that allow for MR analyses with increased statistical power will enable further parsing of the independent effects of OSA on AF with the multivariable MR methodology.

\subsection{Strengths and Limitations}

Our study provides evidence supporting previous RCTs with the methodological strengths of the MR design, which addresses key limitations of conventional observational epidemiological studies. Principally, independent allele distribution at conception distributes confounders equally, analogous to a 'natural' RCT. Additionally, disease pathogenesis is unlikely to alter germline genotype, rendering associations between genotype and disease outcome less vulnerable to reverse causality [19]. This is particularly important as, following the establishment of a proarrhythmogenic atrial substrate, AF perpetuates further AF, complicating exposure-outcome interactions. Whilst clinically relevant causal effects can only be demonstrated in adequately powered and well-conducted RCTs, MR has previously predicted late-stage therapeutic failure in a phase III cardiovascular trial, where classical observational studies suggested promise [42]. Taken together with the RCTs of CPAP in AF [12,13], the present study provides further evidence to support the recently downgraded IIb recommendation of considering OSA management in patients with AF [1].

Using a GWAS for snoring as a proxy for genetically determined SDB and extrapolating to OSA is a limitation of this study. There are a few reasons why this approach was chosen. In the GWAS for snoring performed by Campos et al. [23], the trait that showed the highest genetic correlation with snoring was OSA $\left(\mathrm{rG}=0.78, p=3 \times 10^{-5}\right)$, indicating a strong association between the traits. This genetic correlation remained significant follow- 
ing sensitivity analysis adjusting for BMI. Similarly, significant genetic correlations were observed between snoring genes and two other measures of overnight oxyhaemoglobin saturation that are known proxies of sleep-disordered breathing: minimum SpO2 saturation, and percentage of sleep with oxyhaemoglobin saturation under $90 \%$. Using these discovery GWAS summary statistics, Campos et al. [23] subsequently developed and validated a PGS in an independent target sample. Participants in the highest PGS decile had around twice the odds of probable OSA compared with those in the lowest decile, indicating the validity of snoring as a genetic proxy for OSA in this study.

Another limitation is that MR analysis considers associations of lifelong, cumulative genetic risk, which is not equivalent to associations measured in conventional epidemiological studies. This explains why ORs of AF per $1-\mathrm{SD} \mathrm{kg} / \mathrm{m}^{2}$ increase in genetically predicted BMI reported in our study are larger than corresponding estimates in a large, contemporary epidemiological meta-analysis [14]. MR estimates should, therefore, not be extrapolated to infer the magnitude of effect of a clinical intervention. Additionally, summary GWAS data used in this study [22-24] were derived mainly from individuals of European ancestry, which may limit the generalisability of our findings to non-European ancestry populations. Although further study in these populations is warranted, using ethnically homogeneous samples in our analysis reduces the likelihood of associations between genetic variant and outcome being confounded by hidden population structure. Finally, a degree of overlap existed between the SDB and AF cohort, with a maximum estimated overlap of $78 \%$ and, therefore, a resultant risk of bias of $\sim 7.8 \%$. However, this would bias toward a Type 1 rather than Type 2 error and, therefore, it should not produce bias in this negative study finding.

\section{Conclusions}

In summary, our MR study explored the relationships between SDB, BMI and AF. Our results support previous observational and RCT evidence that BMI is likely to be causally related to the development of $\mathrm{AF}$, while adding that these effects are likely independent of SDB. Our study suggests that an independent causal pathway between SDB, in general, and AF is unlikely. However, we cannot exclude that the more severe forms of SDB, such as OSA, are independently causally associated with AF. The RCTs currently recruiting to explore the effects of CPAP therapy on AF outcomes should further clarify the role of OSA management in patients with AF and inform clinical guidelines.

Supplementary Materials: The following are available online at https:/ / www.mdpi.com/article / 10.3390/genes13010104/s1, Table S1: MR results investigating the effect of SDB and BMI on the primary outcome of AF, Table S2: SNP MR estimates in the analysis of SDB and AF, Table S3: SNP MR estimates in the analysis of BMI and AF.

Author Contributions: Conceptualization, M.A., R.K.R., K.H.K.P. and F.S.N.; methodology, E.A.W.S., D.K.R. and D.G.; formal analysis, M.A., E.A.W.S. and D.K.R.; writing-original draft preparation, M.A. and R.K.R.; writing—review and editing, E.A.W.S., K.H.K.P., D.K.R., D.G. and F.S.N.; supervision, E.A.W.S., D.G. and F.S.N.; project administration, M.A. and R.K.R.; funding acquisition, M.A., D.K.R., D.G. and F.S.N. All authors have read and agreed to the published version of the manuscript.

Funding: This study was supported by the British Heart Foundation (RG/16/3/32175 for FSN, RE/18/4/34215 for DG), the National Institute for Health Research (Imperial NIHR Biomedical Research Centre funding for FSN and KHKP, NIHR Academic Foundation Training Programme for MA, NIHR Clinical Lectureship for DG, NIHR Cambridge BRC funding for EAWS) and the George's Academic Training Small Grant Fund (for DKR).

Institutional Review Board Statement: The study was conducted according to the guidelines of the Declaration of Helsinki. Ethical review and approval were waived for this study, as all data used for this study are publicly available and their original studies are cited. All these studies obtained relevant participant consent and ethical approval.

Informed Consent Statement: All data used for this study are publicly available and their original studies are cited. All these studies obtained relevant participant consent. 
Data Availability Statement: All data used for this study are publicly available and their original studies are cited.

Conflicts of Interest: D.G. is employed part-time by Novo Nordisk. All other co-authors have no relevant disclosures, and all authors declare there are no conflicts of interest in relation to this work.

\section{References}

1. Hindricks, G.; Potpara, T.; Dagres, N.; Arbelo, E.; Bax, J.J.; Blomström-Lundqvist, C.; Boriani, G.; Castella, M.; Dan, G.-A.; Dilaveris, P.E.; et al. 2020 ESC Guidelines for the diagnosis and management of atrial fibrillation developed in collaboration with the European Association for Cardio-Thoracic Surgery (EACTS): The Task Force for the diagnosis and management of atrial fibrillation of the European Society of Cardiology (ESC) Developed with the special contribution of the European Heart Rhythm Association (EHRA) of the ESC. Eur. Heart J. 2021, 42, 373-498.

2. Linz, D.; Woehrle, H.; Bitter, T.; Fox, H.; Cowie, M.R.; Böhm, M.; Oldenburg, O. The importance of sleep-disordered breathing in cardiovascular disease. Clin. Res. Cardiol. 2015, 104, 705-718. [CrossRef]

3. Peppard, P.E.; Young, T.; Barnet, J.H.; Palta, M.; Hagen, E.W.; Hla, K.M. Increased prevalence of sleep-disordered breathing in adults. Am. J. Epidemiol. 2013, 177, 1006-1014. [CrossRef] [PubMed]

4. Gami, A.S.; Pressman, G.; Caples, S.M.; Kanagala, R.; Gard, J.J.; Davison, D.E.; Malouf, J.F.; Ammash, N.M.; Friedman, P.A.; Somers, V.K. Association of atrial fibrillation and obstructive sleep apnea. Circulation 2004, 110, 364-367. [CrossRef]

5. Holmqvist, F.; Guan, N.; Zhu, Z.; Kowey, P.R.; Allen, L.A.; Fonarow, G.; Hylek, E.M.; Mahaffey, K.W.; Freeman, J.V.; Chang, P.; et al. Impact of obstructive sleep apnea and continuous positive airway pressure therapy on outcomes in patients with atrial fibrillation-Results from the Outcomes Registry for Better Informed Treatment of Atrial Fibrillation (ORBIT-AF). Am. Heart J. 2015, 169, 647-654.e2. [CrossRef] [PubMed]

6. Cadby, G.; McArdle, N.; Briffa, T.; Hillman, D.R.; Simpson, L.; Knuiman, M.; Hung, J. Severity of OSA is an independent predictor of incident atrial fibrillation hospitalization in a large sleep-clinic cohort. Chest 2015, 148, 945-952. [CrossRef]

7. $\quad$ Li, L.; Wang, Z.; Li, J.; Ge, X.; Guo, L.-Z.; Wang, Y.; Guo, W.-H.; Jiang, C.-X.; Ma, C.-S. Efficacy of catheter ablation of atrial fibrillation in patients with obstructive sleep apnoea with and without continuous positive airway pressure treatment: A meta-analysis of observational studies. Europace 2014, 16, 1309-1314. [CrossRef]

8. Gami, A.S.; Hodge, D.O.; Herges, R.M.; Olson, E.J.; Nykodym, J.; Kara, T.; Somers, V.K. Obstructive sleep apnea, obesity, and the risk of incident atrial fibrillation. J. Am. Coll. Cardiol. 2007, 49, 565-571. [CrossRef] [PubMed]

9. Mehra, R.; Stone, K.L.; Varosy, P.D.; Hoffman, A.R. Nocturnal Arrhythmias across a spectrum of obstructive and central sleepdisordered breathing in older men: Outcomes of sleep disorders in older men (MrOS sleep) study. Arch. Intern. Med. 2009, 169, 1147-1155. [CrossRef]

10. Yaeger, A.; Keenan, B.T.; Cash, N.R.; Parham, T.; Frankel, D.S.; Deo, R.; Schaller, R.D.; Santangeli, P.; Nazarian, S.; Supple, G.E.; et al. Impact of a nurse-led limited risk factor modification program on arrhythmia outcomes in patients with atrial fibrillation undergoing catheter ablation. J. Cardiovasc. Electrophysiol. 2020, 31, 423-431. [CrossRef]

11. Srivali, N.; Chahal, A.C.; Mansukhani, M.P.; Mandrekar, J.; Somers, V.K.; Caples, S.M. The Effect of Positive Airway Pressure Treatment of Obstructive and Central Sleep Apnea on the Recurrence of Atrial Fibrillation/Flutter Postintervention. J. Clin. Sleep Med. 2019, 15, 1459-1468. [CrossRef] [PubMed]

12. Caples, S.M.; Mansukhani, M.P.; Friedman, P.A.; Somers, V.K. The impact of continuous positive airway pressure treatment on the recurrence of atrial fibrillation post cardioversion: A randomized controlled trial. Int. J. Cardiol. 2019, 278, 133-136. [CrossRef] [PubMed]

13. Traaen, G.M.; Aakerøy, L.; Hunt, T.-E.; Øverland, B.; Bendz, C.; Sande, L.; Aakhus, S.; Fagerland, M.W.; Steinshamn, S.; Anfinsen, O.-G.; et al. Effect of Continuous Positive Airway Pressure on Arrhythmia in Atrial Fibrillation and Sleep Apnea: A Randomized Controlled Trial. Am. J. Respir. Crit. Care Med. 2021, 204, 573-582. [CrossRef] [PubMed]

14. Wong, C.X.; Sullivan, T.; Sun, M.T.; Mahajan, R.; Pathak, R.K.; Middeldorp, M.; Twomey, D.; Ganesan, A.N.; Rangnekar, G.; Roberts-Thomson, K.C.; et al. Obesity and the Risk of Incident, Post-Operative, and Post-Ablation Atrial Fibrillation: A Meta-Analysis of 626,603 Individuals in 51 Studies. JACC Clin. Electrophysiol. 2015, 1, 139-152. [CrossRef]

15. Wong, C.X.; Ganesan, A.N.; Selvanayagam, J.B. Epicardial fat and atrial fibrillation: Current evidence, potential mechanisms, clinical implications, and future directions. Eur. Heart J. 2017, 38, 1294-1302. [CrossRef]

16. Romero-Corral, A.; Caples, S.M.; Lopez-Jimenez, F.; Somers, V.K. Interactions between obesity and obstructive sleep apnea: Implications for treatment. Chest 2010, 137, 711-719. [CrossRef]

17. Newman, A.B.; Foster, G.; Givelber, R.; Nieto, F.J.; Redline, S.; Young, T. Progression and regression of sleep-disordered breathing with changes in weight: The Sleep Heart Health Study. Arch. Intern. Med. 2005, 165, 2408-2413. [CrossRef]

18. Phillips, B.G.; Hisel, T.M.; Kato, M.; Pesek, C.A.; Dyken, M.; Narkiewicz, K.; Somers, V.K. Recent weight gain in patients with newly diagnosed obstructive sleep apnea. J. Hypertens. 1999, 17, 1297-1300. [CrossRef] [PubMed]

19. Lawlor, D.A.; Harbord, R.M.; Sterne, J.A.C.; Timpson, N.; Smith, G.D. Mendelian randomization: Using genes as instruments for making causal inferences in epidemiology. Stat. Med. 2008, 27, 1133-1163. [CrossRef] [PubMed]

20. Chen, W.; Cai, X.; Yan, H.; Pan, Y. Causal Effect of Obstructive Sleep Apnea on Atrial Fibrillation: A Mendelian Randomization Study. J. Am. Heart Assoc. 2021, e022560, Epub ahead of print. [CrossRef] 
21. STROBE-MR Steering Group: STROBE-MR: Guidelines for strengthening the reporting of Mendelian randomization studies. PeerJ 2019. [CrossRef]

22. Yengo, L.; Sidorenko, J.; Kemper, K.E.; Zheng, Z.; Wood, A.R.; Weedon, M.; Frayling, T.; Hirschhorn, J.; Yang, J.; Visscher, P.M.; et al. Meta-analysis of genome-wide association studies for height and body mass index in $~ 700000$ individuals of European ancestry. Hum. Mol. Genet. 2018, 27, 3641-3649. [CrossRef] [PubMed]

23. Campos, A.I.; García-Marín, L.M.; Byrne, E.M.; Martin, N.G.; Cuéllar-Partida, G.; Rentería, M.E. Insights into the aetiology of snoring from observational angd genetic investigations in the UK Biobank. Nat. Commun. 2020, 11, 817. [CrossRef]

24. Roselli, C.; Chaffin, M.D.; Weng, L.-C.; Aeschbacher, S.; Ahlberg, G.; Albert, C.M.; Almgren, P.; Alonso, A.; Anderson, C.D.; Aragam, K.G.; et al. Multi-ethnic genome-wide association study for atrial fibrillation. Nat. Genet. 2018, 50, 1225-1233. [CrossRef]

25. Hemani, G.; Zheng, J.; Elsworth, B.; Wade, K.H.; Haberland, V.; Baird, D.; Laurin, C.; Burgess, S.; Bowden, J.; Langdon, R.; et al. The MR-Base platform supports systematic causal inference across the human phenome. Elife 2018, 7, e34408. [CrossRef] [PubMed]

26. Brion, M.J.; Shakhbazov, K.; Visscher, P.M. Calculating statistical power in Mendelian randomization studies. Int. J. Epidemiol. 2013, 42, 1497-1501. [CrossRef] [PubMed]

27. Burgess, S.; Davey Smith, G.; Davies, N.M.; Dudbridge, F.; Gill, D.; Glymour, M.M.; Hartwig, F.P.; Holmes, M.V.; Minelli, C.; Relton, C.L.; et al. Guidelines for performing Mendelian randomization investigations. Wellcome Open Res. 2020, 4, 186. [CrossRef] [PubMed]

28. Bowden, J.; Davey Smith, G.; Burgess, S. Mendelian randomization with invalid instruments: Effect estimation and bias detection through Egger regression. Int. J. Epidemiol. 2015, 44, 512-525. [CrossRef]

29. Bowden, J.; Smith, G.D.; Haycock, P.C.; Burgess, S. Consistent Estimation in Mendelian Randomization with Some Invalid Instruments Using a Weighted Median Estimator. Genet. Epidemiol. 2016, 40, 304-314. [CrossRef]

30. Verbanck, M.; Chen, C.-Y.; Neale, B.; Do, R. Detection of widespread horizontal pleiotropy in causal relationships inferred from Mendelian randomization between complex traits and diseases. Nat. Genet. 2018, 50, 693-698. [CrossRef]

31. Slob, E.A.W.; Burgess, S. A comparison of robust Mendelian randomization methods using summary data. Genet. Epidemiol. 2020, 44, 313-329. [CrossRef] [PubMed]

32. Burgess, S.; Thompson, S.G. Interpreting findings from Mendelian randomization using the MR-Egger method. Eur. J. Epidemiol. 2017, 32, 377-389. [CrossRef] [PubMed]

33. Rees, J.M.B.; Wood, A.M.; Dudbridge, F.; Burgess, S. Robust methods in Mendelian randomization via penalization of heterogeneous causal estimates. PLoS ONE 2019, 14, e0222362. [CrossRef] [PubMed]

34. Yavorska, O.O.; Burgess, S. MendelianRandomization: An R package for performing Mendelian randomization analyses using summarized data. Int. J. Epidemiol. 2017, 46, 1734-1739. [CrossRef]

35. Chatterjee, N.A.; Giulianini, F.; Geelhoed, B.; Lunetta, K.L.; Misialek, J.R.; Niemeijer, M.N.; Rienstra, M.; Rose, L.M.; Smith, A.V.; Arking, D.E.; et al. Genetic Obesity and the Risk of Atrial Fibrillation: Causal Estimates from Mendelian Randomization. Circulation 2017, 135, 741-754. [CrossRef]

36. Larsson, S.C.; Bäck, M.; Rees, J.M.B.; Mason, A.M.; Burgess, S. Body mass index and body composition in relation to 14 cardiovascular conditions in UK Biobank: A Mendelian randomization study. Eur. Heart J. 2020, 41, 221-226. [CrossRef] [PubMed]

37. Abed, H.S.; Samuel, C.S.; Lau, D.H.; Kelly, D.J.; Royce, S.G.; Alasady, M.; Mahajan, R.; Kuklik, P.; Zhang, Y.; Brooks, A.G.; et al. Obesity results in progressive atrial structural and electrical remodeling: Implications for atrial fibrillation. Heart Rhythm 2013, 10, 90-100. [CrossRef]

38. Abed, H.S.; Wittert, G.A.; Leong, D.P.; Shirazi, M.G.; Bahrami, B.; Middeldorp, M.E.; Lorimer, M.F.; Lau, D.H.; Antic, N.A.; Brooks, A.G.; et al. Effect of weight reduction and cardiometabolic risk factor management on symptom burden and severity in patients with atrial fibrillation: A randomized clinical trial. JAMA 2013, 310, 2050-2060. [CrossRef] [PubMed]

39. Rienstra, M.; Hobbelt, A.H.; Alings, M.; Tijssen, J.G.P.; Smit, M.D.; Brügemann, J.; Geelhoed, B.; Tieleman, R.G.; Hillege, H.L.; Tukkie, R.; et al. Targeted therapy of underlying conditions improves sinus rhythm maintenance in patients with persistent atrial fibrillation: Results of the RACE 3 trial. Eur. Heart J. 2018, 39, 2987-2996. [CrossRef] [PubMed]

40. Iwasaki, Y.-K.; Shi, Y.; Benito, B.; Gillis, M.-A.; Mizuno, K.; Tardif, J.-C.; Nattel, S. Determinants of atrial fibrillation in an animal model of obesity and acute obstructive sleep apnea. Heart Rhythm. 2012, 9, 1409-1416.e1. [CrossRef]

41. Smith, G.D.; Phillips, A. Declaring independence: Why we should be cautious. J. Epidemiol. Community Health 1990, 44, 257-258. [CrossRef] [PubMed]

42. Polfus, L.M.; Gibbs, R.A.; Boerwinkle, E. Coronary Heart Disease and Genetic Variants with Low Phospholipase A2 Activity. N. Engl. J. Med. 2015, 372, 295-296. [CrossRef] [PubMed] 